International Journal of Engineering \&Technology, $7(1.7)(2018)$ 27-33
International Journal of Engineering \& Technology
WPC
Website: www.sciencepubco.com/index.php/IJET
Research paper

\title{
Secure data in cloud with multimodal key generation
}

\author{
P Selvarani $^{1 *}, \mathbf{N}$ Malarvizhi ${ }^{2}$ \\ ${ }^{1}$ Research Scholar, Department of Computer Science and Engineering, School of Computing, Vel Tech Rangarajan \\ Dr. Sagunthala R\&D Institute of Science and Technology, Avadi, Chennai-62, TamilNadu, India \\ ${ }^{2}$ Professor, Department of Computer Science and Engineering, School of Computing, Vel Tech Rangarajan \\ Dr. Sagunthala R\&D Institute of Science and Technology, Avadi, Chennai-62, TamilNadu, India \\ *Corresponding author E-mail: selvarani.meena@gmail.com
}

\begin{abstract}
Data Security is the Major problem in Cloud Computing. In order to overcome the data security problem the proposed technique utilizes effective data storage using biometric-based cryptographic authentication to support the user authentication for the cloud environment. For user authentication here we are considering iris and fingerprint. Initially the feature values are extracted from the iris and fingerprint using local binary pattern and Minutiae extraction respectively. Local binary pattern operator works with the eight neighbors of a pixel, using the value of this center pixel as a threshold. Minutiae points are the major features of a fingerprint image and are used in the matching of fingerprints. These minutiae points are used to determine the uniqueness of a fingerprint image. Based on that the proposed feature values are extracted from the iris and fingerprint image. In order to improve the security, the suggested technique utilizes the optimal features. For selecting the optimal features hybrid particle swarm optimization and genetic algorithm (HPSOGA) is utilized. Particle swarm optimization (PSO) is a population based stochastic optimization technique. The system is initialized with a population of random solutions and searches for optima by updating generations. In PSO, the potential solutions, called particles, fly through the problem space by following the current optimum particles. Genetic Algorithms (GAs) are adaptive heuristic search algorithm based on the evolutionary ideas of natural selection and genetics. In our proposed method these two optimization algorithm is hybrid for more secure. From the optimization algorithm the suggested technique selects the optimal features. and then the optimal features are used to encrypt the input data. For encryption and decryption, the proposed technique utilizes Triple DES algorithm. Finally the encrypted data is stored in cloud. The performance of the proposed technique is evaluated in terms of encryption and decryption time, memory utilization and overall execution time. Our proposed data storage using biometric-based authentication is implemented with the help of Cloud simulator in the working platform of java.
\end{abstract}

Keywords: Multimodal Bio Cryptographic Authentication; Local Binary Pattern; Hybrid Particle Swarm Optimization with Genetic Algorithm; Triple DES Algorithm; Cloud Storage Environment.

\section{Introduction}

Cloud computing is an emerging computing technology that uses the internet and central remote servers to maintain data and application. Data security becomes more and more important in cloud computing. [1] because hackers can hack the data during data transfer. Hacking means unauthorized user access the data without data owner authorization. [2] So authorized owner will lose billions of dollars due to illegal activities like copying creating and destroying the data without data owner authorization. So it is important to secure the cloud data. Figure 1 Represents Hackers hack the data.

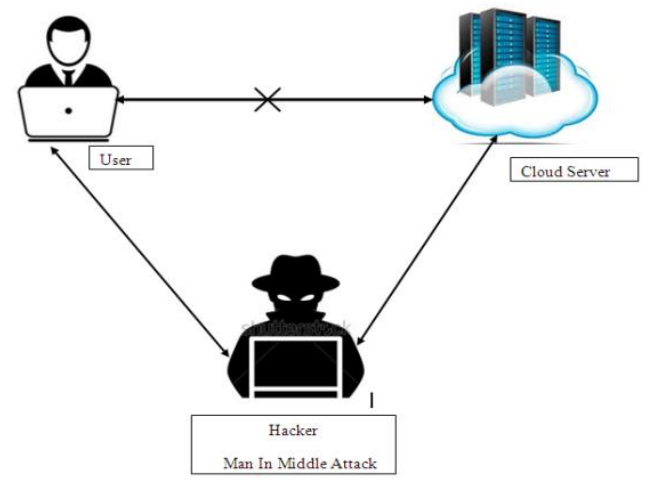

Fig. 1: Represents Meet in Middle Attack.

The user authentication, which is main part of the cloud computing, determines only the authorized user is to access the data. The best way is to encrypt the data before send it to a third party. The problem with storing the data in cloud environment using password system used as a key to encrypt the data, it is not secured, forgotten and easily stolen. [3]. To overcome this problem multimodal bio cryptographic technique [4] can be used to support the user authentication in cloud environment because it is more relia- 
ble than password based system, stable, not forgotten, don't stolen, forgery, copied, shared and distributed etc.,

\subsection{Overall process}

User authentication here we are considering Fingerprint and iris. Figure 2 represents overall process of our research work. Initially User has given input to the fingerprint image and iris image. Feature can be extracted from the fingerprint and iris image using Local Binary Pattern. Generating feature value can be combined and it can be given input to the Hybrid Genetic and Particle swarm optimization algorithm for finding best solution.

The best solution can be act as a key to encrypt and decrypt the data using Triple DES algorithm. Finally Stored in Cloud Environment. So the intruder cannot be able to access the data.

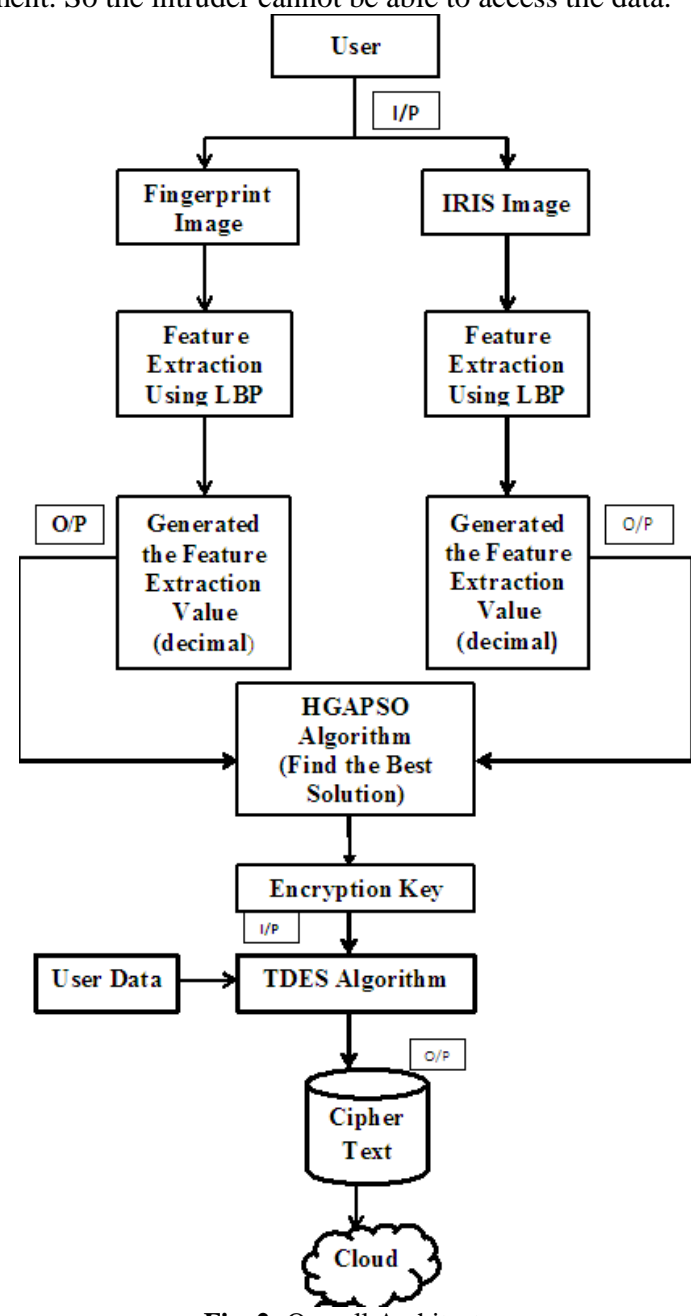

Fig. 2: Overall Architecture.

\subsection{Local binary pattern}

Local binary pattern operator works with the eight neighbors of a pixel, using the value of this center pixel as a threshold.

Calculate the Fingerprint and Iris Feature Extraction values using LBP. Each pixel Find its LBP. Compare the center pixel value of its neighbor. Center pixel value is greater than the neighbor value becomes 0 other wise 1. [5]. Likewise all the pixel value can be calculated by using LBP. Finally Binary value converted into decimal value. Same process can be used as extracting the feature value of Iris. Figure 3 represents Process of Local Binary Pattern.

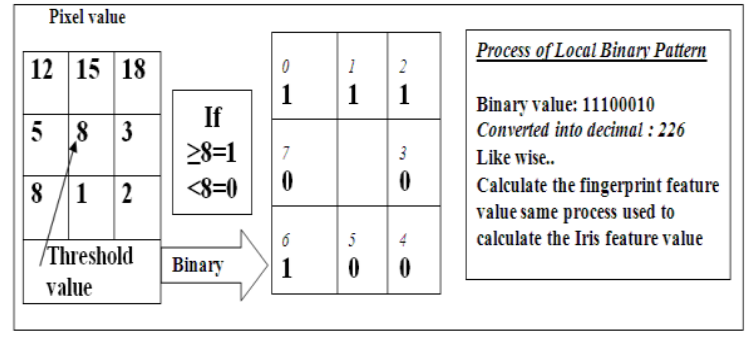

Fig. 3: Process of Local Binary Pattern.

Figure 4 represents Fingerprint feature Values, and Figure 5 represents Iris feature Values by using Local Binary Pattern.

\section{Fingerprint feature value using LBP}

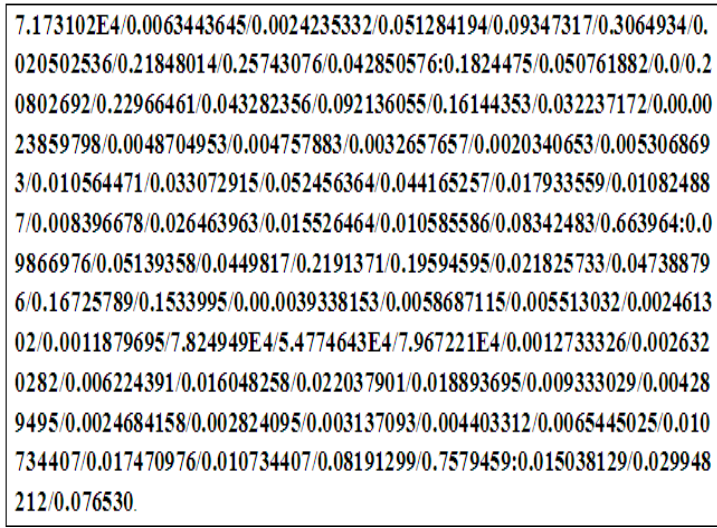

Fig. 4: Fingerprint Feature Value Using LBP.

\section{Iris feature value using LBP}

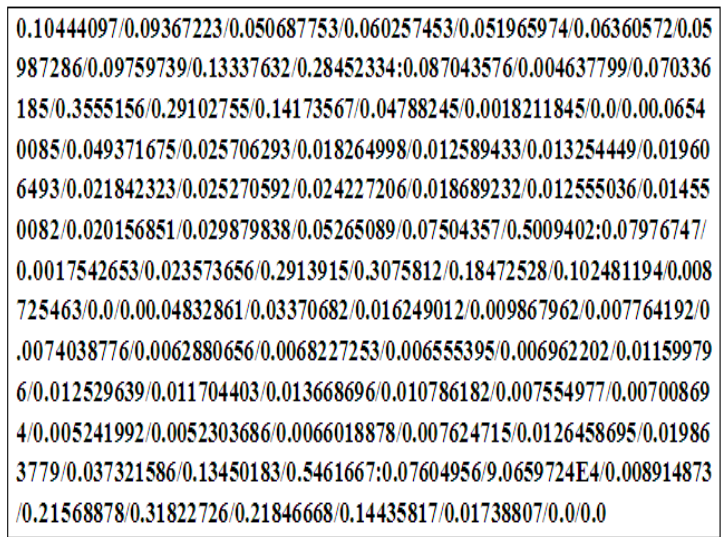

Fig. 5: Iris Feature Value Using LBP.

\subsection{Combined feature value of fingerprint and iris with HGAPSO}

Extracted value of Fingerprint and Iris can be combined and it has been given input to the hybrid Genetic Algorithm[6] and Particle swarm optimization algorithm [7] to find the best solution by using cross over mutation technique. Figure 6 Represents Process of HGAPSO Algorithm. 


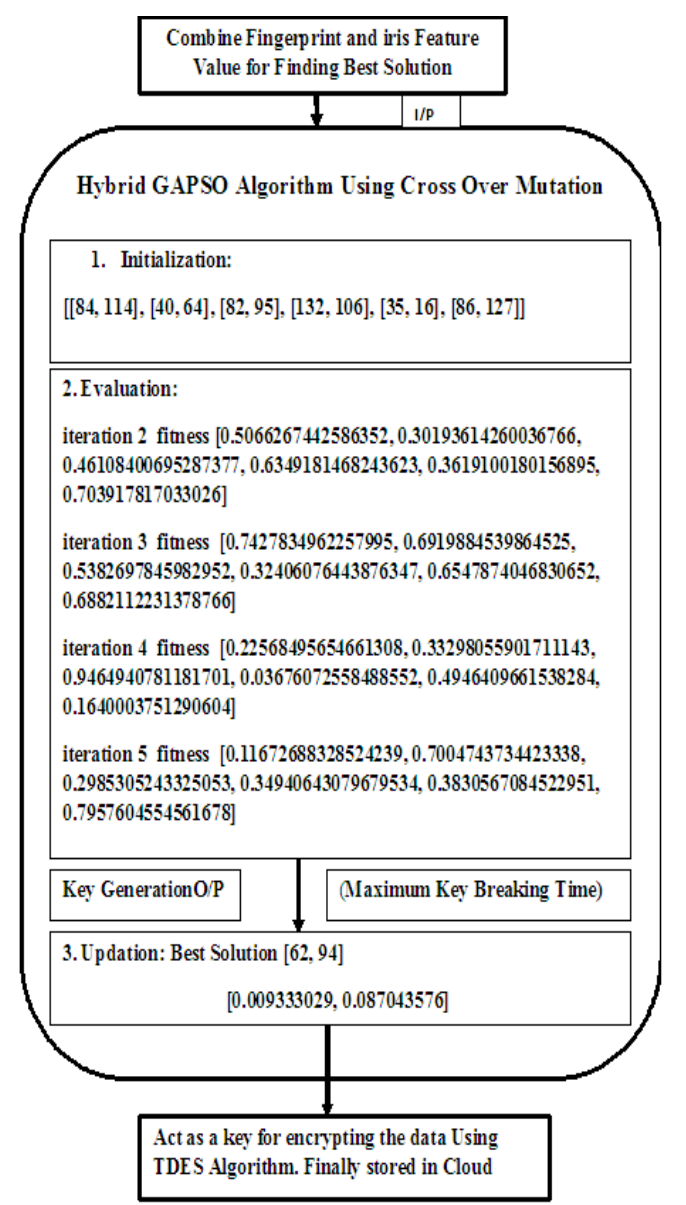

Fig. 6: Process of HGAPSO Algorithm.

To improve the optimization performance can be used as Hybrid GA+PSO as more secure by using, (Figure 7 Represents) Cross over Mutation Technique as Selection, Recombination and Mutation.

\subsection{Selection}

Replicate the most successful solutions found in a population at a rate proximal to their relative quantity.

\subsection{Recombination}

Decomposes two distinct solutions and then randomly mixes their parts to form novel solution.

\subsection{Mutation}

Randomly perturbs a candidate's solution.

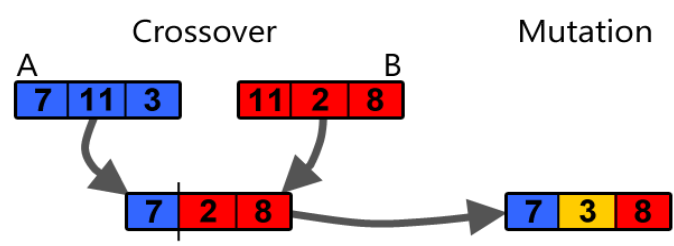

Fig. 7: Cross over Mutation.

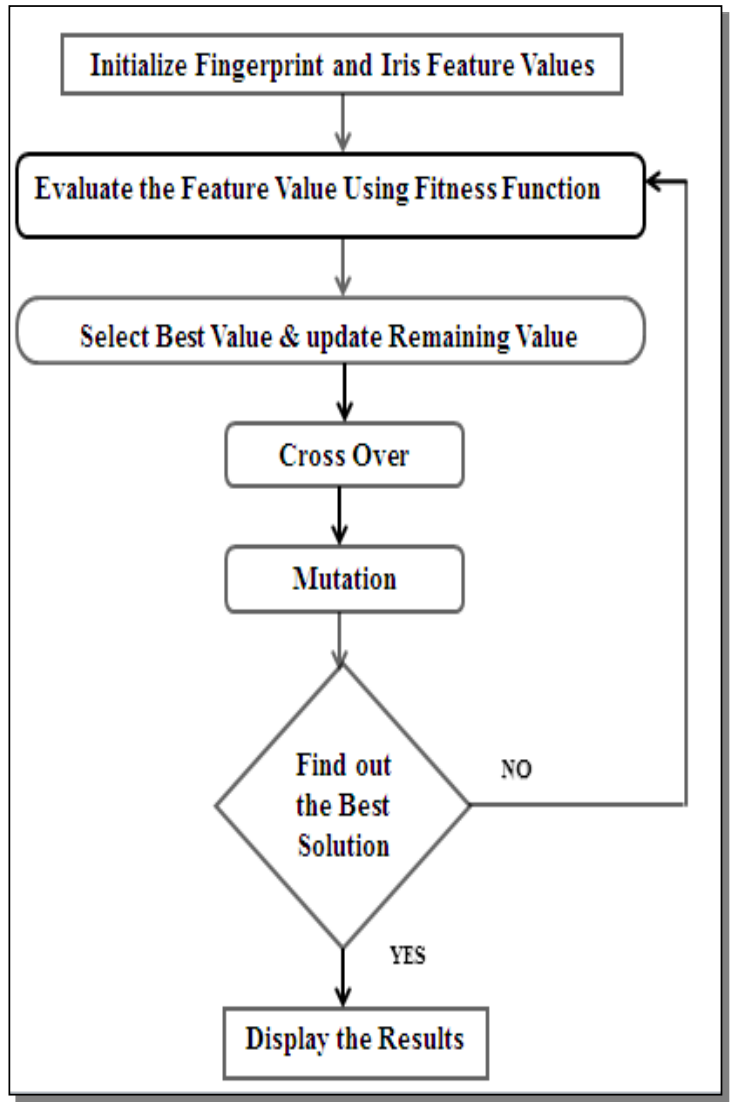

Fig. 8: Flowchart of HGAPSO Algorithm.

\subsection{HGAPSO Algorithm}

\begin{tabular}{|c|c|}
\hline $\begin{array}{l}\text { HGAPSO Algorithm } \\
\text { int it=1; iteraion=10 } \\
\text { Input=FP,Iris } \\
\text { For } x=1 \text { to6 } \\
\text { Find best fgp(x) } \\
\text { Velocity update(x) } \\
\text { solution update(x) } \\
\text { pso.crossover_mutuation } \\
\text { (x,input.size(0); } \\
\text { While(it }<\text { iteration) } \\
\{ \\
\text { Fitt=pso.findfitness(inputt, } x) \text {; } \\
\text { Find newgp(x); } \\
\text { Velocity update(x); } \\
\text { Solution update(x); } \\
\text { it++; } \\
\text { System.out.println("iteration"+it+"fitness"+fi } \\
\text { ft); } \\
\text { \} } \\
\text { Sytem.out.println("best solution"+gb), } \\
\text { ("best solution"+gb) }\end{array}$ & $\begin{array}{l}\text { Where } \\
\text { Input } \rightarrow \text { Fingerprint and Iris Feature } \\
\quad \text { Extraction Values } \\
\text { it } \rightarrow \text { initialization } \\
\mathrm{x} \rightarrow \text { List of array } \\
\text { gp } \rightarrow \text { best value } \\
\text { Velocity update }(\mathrm{x})\end{array}$ \\
\hline
\end{tabular}

Fig. 9: HAPSO Algorithm.

Initialize Fingerprint and Iris Feature Extraction Values. Evaluate the Feature Values using Fitness Function.

(Task: Maximum Key Breaking Time

Choose the Best Value and Update the Remaining Value.

Compare the initialized value with updated value and also calculate the fitness function

Repeat the iteration, till to find out the best solution.

Finally stop the iteration. Best solution act as a key for encrypting the data using TDES Algorithm.

Fig. 10: Process of HGAPSO.

To find out the best solution key is 0.0221135095 used as a key for encrypting and decrypting data using Triple DES algorithm. 


\begin{tabular}{|c|c|c|c|c|c|}
\hline Algorithm & Developed by & Manners & Method & Implementation & Preference \\
\hline $\begin{array}{c}\text { PSO } \\
\text { Particle } \\
\text { Swarm } \\
\text { Optimization }\end{array}$ & $\begin{array}{l}\text { Dr. Ebhart and } \\
\text { Dr. Kenady in } \\
1995\end{array}$ & $\begin{array}{l}\text { Naturally } \\
\text { behavior of bird } \\
\text { flocking and fish } \\
\text { schooling for } \\
\text { finding food } \\
\text { source. }\end{array}$ & $\begin{array}{l}\text { Velocity } \\
\text { Updation } \\
\text { Position } \\
\text { Updation }\end{array}$ & $\begin{array}{l}\text { Simple, easy to } \\
\text { implement, } \\
\text { Computationally } \\
\text { efficient }\end{array}$ & $\begin{array}{l}\text { Aritificial Neural Network Training, } \\
\text { FuzzySystemcontrol,Telecommunications, } \\
\text { DataMining,Combinotorial Optimization, Power } \\
\text { Systems, Signal processing and Many others }\end{array}$ \\
\hline $\begin{array}{c}\text { GA } \\
\text { Genetic } \\
\text { Algorithm }\end{array}$ & $\begin{array}{c}\text { John Holland in } \\
1975\end{array}$ & $\begin{array}{l}\text { Genetic behavior } \\
\text { of Parent and } \\
\text { Child. }\end{array}$ & $\begin{array}{l}\text { Cross } \\
\text { Over and } \\
\text { Mutation }\end{array}$ & $\begin{array}{l}\text { Easy to Exploit, } \\
\text { support-multi } \\
\text { objective optimization }\end{array}$ & $\begin{array}{l}\text { Bioinformatics, phylogenetic, computational } \\
\text { science, engineering, economics, chemistry, } \\
\text { manufacturing, mathematics, physics other fields }\end{array}$ \\
\hline HGAPSO & \multicolumn{5}{|c|}{ Improving the optimization performance it can be used as hybrid and More Secure } \\
\hline $\begin{array}{c}\text { Hybrid } \\
\text { GA+PSO }\end{array}$ & \multicolumn{4}{|c|}{$\begin{array}{l}\text { Both having } \\
\text { Population based Stochastic Optimization Technique } \\
\text { Random Generation } \\
\text { Fitness Function for evaluating purpose }\end{array}$} & $\begin{array}{l}\text { Difference } \\
\text { PSO does not have Genetic operator like Cross } \\
\text { over and Mutation. } \\
\text { But they also are having Memory. }\end{array}$ \\
\hline
\end{tabular}

Fig. 11: PSO Vs. GA.

\subsection{Cryptographic technique}

William Stallings proposed cryptographic technique for the purpose of data security, in the concept of plaintext can be converted to cipher text called encryption and for the reverse process of decryption. There are two types of Cryptographic Algorithm. Symmetric algorithm and Asymmetric algorithm. [8].Same key can be used for symmetric and different key can be used for asymmetric algorithm. Symmetric key algorithm such as DES, AES, 3DES, Blowfish etc., and Asymmetric key algorithm such as RSA, Diffie-helman key exchange etc., for more security purpose

Triple DES algorithm can be used for data encryption and decryption.

\subsection{Triple DES algorithm}

Triple DES algorithm uses three iterations of common DES cipher. It receives a secret 168-bit key, which is divided into three 56-bit keys. Encryption using the first secret key Decryption using the second secret key Encryption using the third secret key.

Encryption: c = E3 (D2 (E1 (m)))

Decryption=D1 (E2 (D3 (c)))

In this research work find out the best solution 0.0221135095 (This value derived from fingerprint and iris).It can be act as a key for encrypting and decrypting the data. So the intruder cannot be able to access the encrypted data why because particular portions of fingerprint and iris value can be optimized. So it is more secured.

\begin{tabular}{|l|l|l|}
\hline Factor & DES & 3DES \\
\hline Developed & IBM in 1975 & IBM in 1978 \\
\hline Description & $\begin{array}{l}\text { Block Cipher, } \\
\text { Encrypt- 64 bit data } \\
\text { block. } \\
\text { Fixed Length 64 bit key } \\
\text { (only 56 bit key used for } \\
\text { encryption) }\end{array}$ & $\begin{array}{l}\text { Apply DES in 3 } \\
\text { times in a row using } \\
\text { three different keys. } \\
\text { key size of 168 and } \\
112 \text { bits }\end{array}$ \\
\hline Block size & 64 bits & 64 bits \\
\hline Key Size & 56 bits & $\begin{array}{l}168 \text { bits (3-key) } \\
\text { 112 bits (2-key) }\end{array}$ \\
\hline Possible Keys & $2^{\wedge}$ 56 & 2^112 \\
\hline *Keys & 1 & 3 \\
\hline $\begin{array}{l}\text { Rounds run } \\
\text { through algorithm }\end{array}$ & 16 & 48 \\
\hline Cipher type & Symmetric Block & Symmetric Block \\
\hline $\begin{array}{l}\text { Algorithm } \\
\text { Structure }\end{array}$ & Feistal Network & Feistal Network \\
\hline security & week & Brute force Attack \\
\hline Attack & $\begin{array}{l}\text { Substitution and } \\
\text { Permutation }\end{array}$ & $\begin{array}{l}\text { Not yet -Meet in } \\
\text { Middle Attack }\end{array}$ \\
\hline $\begin{array}{l}\text { Encryption } \\
\text { Primitives }\end{array}$ & Permutation \\
\hline $\begin{array}{l}\text { Cryptographic } \\
\text { Primitives }\end{array}$ & $\begin{array}{l}\text { Confusion and } \\
\text { Diffusion }\end{array}$ \\
\hline
\end{tabular}

Fig. 12: Triple DES.

\subsection{Testing}

Table 4 Represents Randomly Testing fingerprint and iris image to find out the attack by using in the existing technique of Particle Swarm Optimization algorithm with proposed technique of Hybrid Genetic with Particle Swarm optimization Algorithm. It can be find our Less Attack by comparing HGAPSO Algorithm. So it can be concluded as HAPSO is better than PSO.

\begin{tabular}{lll}
\hline Images & HGAPSO & PSO \\
\hline & $\begin{array}{l}\text { Best Solution } \\
{[84,50]}\end{array}$ & $\begin{array}{l}\text { Best Solution [78, 11] } \\
{[0.15580185,0.052767564]}\end{array}$ \\
$\begin{array}{l}\text { Fingerprint -109_3.png } \\
\text { Iris-108_6.png }\end{array}$ & {$[0.10444097,0.0017286023]$} & Best Solution [13, 92] \\
$\begin{array}{l}\text { Fingerprint -109_4.png } \\
\text { Iris-108_7.png }\end{array}$ & Best Solution [139, 76] & {$[0.24218619,0.1362495]$} \\
Fingerprint -109_5.png & {$[0.0063461806,0.08490781]$} & Best Solution [67, 125] \\
Iris-109_1.png & Best Solution $[62,34]$ & {$[0.0084936265,0.30564347]$} \\
Fingerprint -109_6.png & {$[0.0071847257,0.017490147]$} & Best Solution [162, 16] \\
Iris-109_2.png & Best Solution [95, 87] & {$[0.32856014,0.07470472$} \\
Fingerprint -109_7.png & {$[0.0054069953,0.06825483]$} & Best Solution [20, 43] \\
Iris-109_3.png & Best Solution $[106,99]$ & {$[0.0023859798,0.021825733]$} \\
\hline
\end{tabular}


Process of Cloud Storage

1. Initialize the cloud sim (version 2.1)

(No of cloud user)

2. Create Data center

(Location of storing the data)

3. Create Mediator

Temporary stored data

4. Create Virtual machine

[VM Description (vmid =0; inage size $(M B)$, ram= vm, memory

(Data storage), Number of cpu (cpu allocation),

vm name, (allocate the virtual machine name)]

and To Add (add another virtual machine)

\section{Create one cloudlet}

Cloud let properties (Eg: id, File, length, file size, etc.,)

Add the cloudlet to the list

Submit cloudlet to the broker

\section{Start the simulation}

Start the simulation ();

Stop the simulation ();

7. Print the results when simulation is over.

Fig. 13: Best solution of HGAPSO with PSO.

\subsection{Process of cloud storage [9]}

Testing Attack HGAPSO with PSO

\begin{tabular}{ll}
\hline $\begin{array}{l}\text { Proposed HGAP- } \\
\text { SO }\end{array}$ & 0.018163456504999995 \\
\hline $\begin{array}{l}\text { Existing PSO } \\
\text { Total Time }\end{array}$ & $\begin{array}{l}0.039866595835000004 \\
1 \text { minute } 16 \text { seconds }\end{array}$ \\
\hline
\end{tabular}

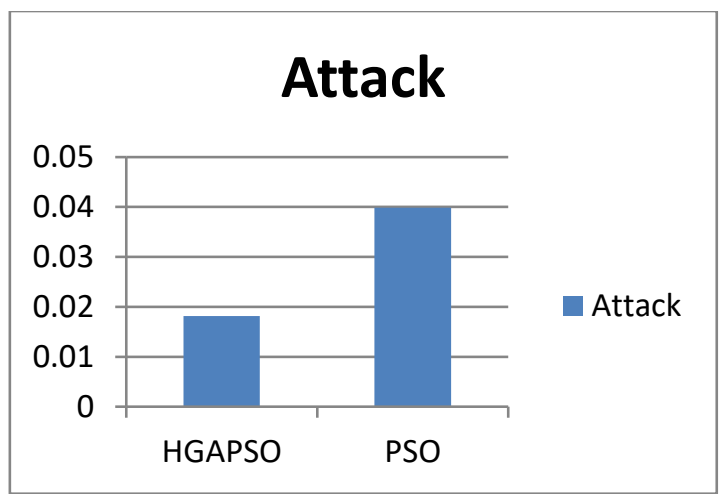

Fig. 14: Less Attack with Comparing PSO. 

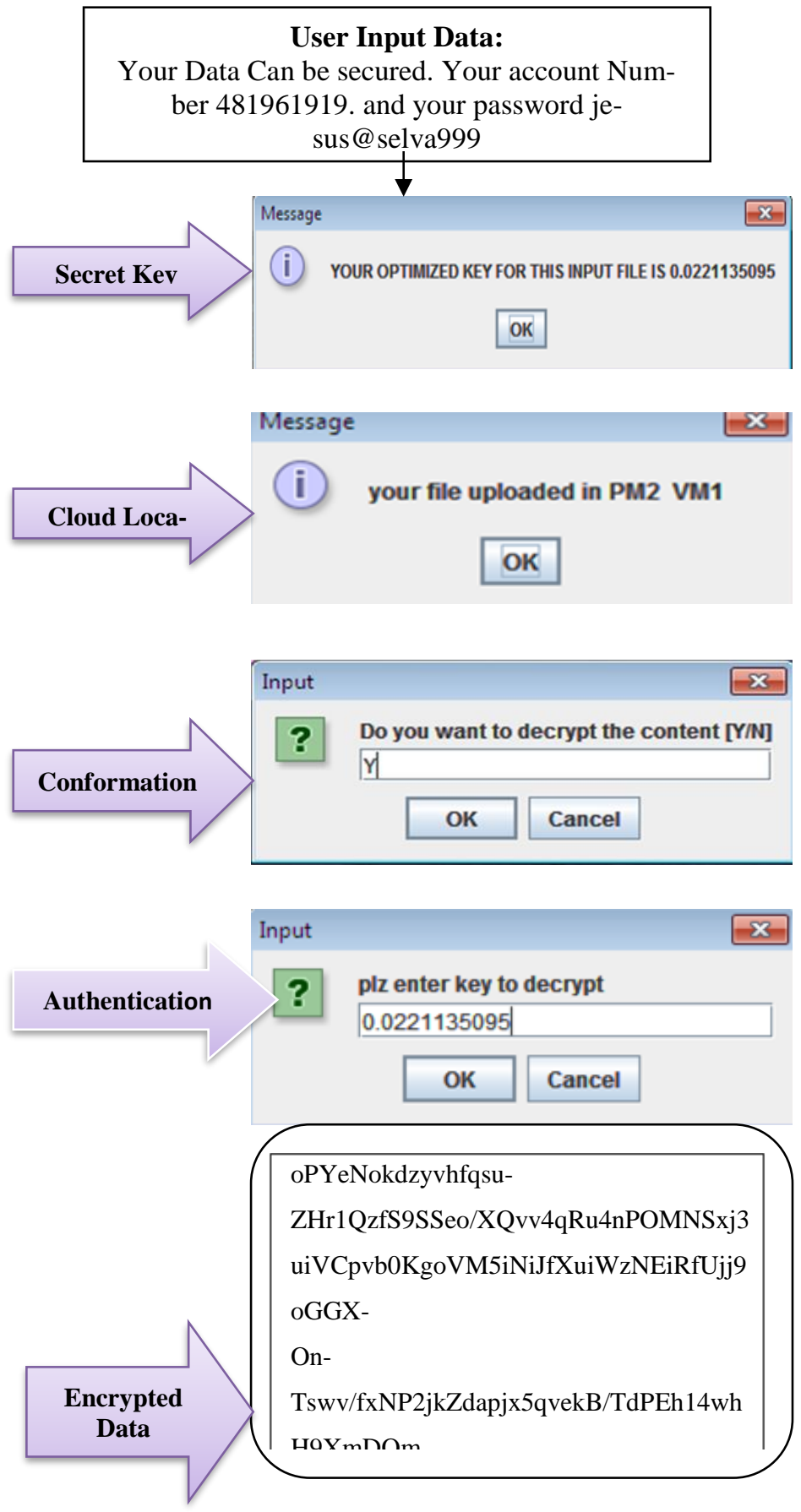

Fig. 15: Process of Encryption and Decryption.

\section{Conclusion}

Derived the Best solution from fingerprint and Iris with the help of LBP, HGAPSO algorithm, Cross over Mutation technique, and Triple DES algorithm. (i) Derived the feature value of fingerprint and Iris using LBP. (ii) To find the Best Solution using HGAPSO algorithm with the help of cross over mutation technique. (iii) To Encrypting the data using Triple DES algorithm and it is stored in cloud environment. So the intruder cannot be able to access the data in cloud environment. In this research work at final stage randomly checking the Fingerprint and iris with the help of Proposed HGAPSO algorithm, and also check with the Existing Particle Swarm optimization algorithm. Comparing both algorithms as per the result wise HGAPSO is better than PSO algorithm. The total successful building time is 1 minute 16 seconds. It can be more secure Less attack and higher data security in cloud.

\section{References}

[1] M. Lori, "Data security in the world of cloud computing," Copublished by the IEEE Computer and reliability Societies, pp. 6164, 2009.

[2] Zhu, Bo; Guang Gong (2011). "MD MITM Attack and Its Applications to GOST, KTANTAN and Hummingbird-2". eCrypt.

[3] S.M. Bellovin and M. Merritt, "Encrypted Key Exchange: Password-Based Protocols Secure Against Dictionary Attacks," Proc. IEEE Symp. Security and Privacy, IEEE CS Press, 1992, pp. 72-84. https://doi.org/10.1109/RISP.1992.213269.

[4] F. Hao, R. Anderson, and J. Daugman. Combining crypto with biometrics effectively. IEEE Transactions on Computers, 55(9):1081- 1088, 2006. https://doi.org/10.1109/TC.2006.138.

[5] Trefný, Jirí, and Jirí Matas."Extended set of local binary patterns for rapid object detection." Proceedings of the Computer Vision Winter Workshop. Vol. 2010.

[6] Goldberg, David (2002). The Design of Innovation: Lessons from and for Competent Genetic Algorithms. Norwell, MA: Kluwer Ac- 
ademic

Publishers. ISBN 978-1402070983

https://doi.org/10.1007/978-1-4757-3643-4.

[7] Kennedy, J. and Eberhart, R., "Particle Swarm Optimization," Proceedings of the IEEE International Conference on Neural Networks, Perth, Australia 1995, pp. 1942-1945. https://doi.org/10.1109/ICNN.1995.488968.

[8] William Stallings, "Cryptography and Network Security: Principles and Practice", Pearson Education/Prentice Hall, 5 th Edition.

[9] K. Yang and X. Jia, "Data storage auditing service in cloud computing: challenges, methods and opportunities," World Wide Web, vol 15, no. 4, pp. 409-428, 2012. https://doi.org/10.1007/s11280-011$\underline{0138-0 .}$. 